|| ISSN(online): 2589-8698 || ISSN(print): 2589-868X ||

International Journal of Medical and Biomedical Studies

Available Online at www.ijmbs.info

NLM (National Library of Medicine ID: 101738825)

Index Copernicus Value 2019: 79.34

Original Research Article

Volume 5, Issue 8; August: 2021; Page No. 323-326

\title{
COVID 19 ASSOCIATED INVASIVE FUNGAL INFECTIONS IN CHURU REGION OF RAJASTHAN
}

\section{Vikas Devra ${ }^{1}$, Varsha Varshney ${ }^{2}$}

${ }^{1}$ Assistant Professor, PanditDeenDayalUpadhyay Medical College Churu, Rajasthan

${ }^{2}$ Consultant Ophthalmologist, Devra Eye Hospital, Churu, Rajasthan

Article Info: Received 22 July 2021; Accepted 14 August 2021

DOI:https://doi.org/10.32553/ijmbs.v5i8.2147

Corresponding author: Vikas Devra

Conflict of interest: No conflict of interest.

\begin{abstract}
Background: COVID 19 or severe acute respiratory syndrome (SARS-CoV-2) evolved as global pandemic since December 2019 when it was first reported in Wuhan, China. Coinfection in patients with COVID-19 has been reported in multiple studies, being bacterial in origin the most frequent; and fungal infection being reported only in severe cases.

Methods: This study was conducted at the department of E.N.T. at PanditDeenDayalUpadhyay Medical College, Churu. Cases presented to E.N.T. OPD with clinical features of invasive rhino-orbital fungal infection for the period of two month from $1^{\text {st }}$ June 2021 were included in the study. Patients were clinically evaluated, HRCT, MRI scans and with KOH mount and culture of involved tissue ere done. Patient's COVID-19 status was ascertained. The clinicopathological association of occurrence of invasive fungal infections in post covid and non-covid patients along with other risk factors like diabetes mellitus, immune compromised state and long-term steroid use was done using statistical methods

Results: We studied 21 patients who attended our OPD with features of invasive fungal infection. Most common presentation was swelling and pain in the cheek region and red eye and swelling around the eye, three patients presented with maxillary swelling with blackish discoloration of teeth. Radiological findings were suggestive of fungal etiology in all patients. KOH mount showed fungal hyphae in 20 cases. Total 20 cases were post COVID status. Old DM was there in 12 patients whereas 9 patients showed new onset hyperglycemia.

Conclusions: COVID-19 is associated with a significant incidence of secondary infections, both bacterial and fungal probably due to immune dysregulation. The widespread use of steroids/monoclonal antibodies/broad-spectrum antibiotics as part of the management protocols against COVID-19 may lead to the exacerbation of preexisting fungal diseases or development of new infection. Treating Physicians should make themselves aware and prepare for the possibility of invasive secondary fungal infections in patients with COVID-19 infection especially in patients with preexisting risk factors and should enable early diagnosis and treatment with the subsequent reduction of mortality and morbidity.
\end{abstract}

Keywords: Coronavirus, COVID-19, mucormycosis, invasive fungal infections

\section{Introduction}

COVID 19 syndrome or severe acute respiratory syndrome coronavirus 2 (SARS-CoV-2) evolved as global pandemic since December 2019 when it was first reported in Wuhan, China.[1] Coinfection in patients with COVID-19 has been reported in multiple studies, being bacterial in origin the most frequent; and fungal infection being reported only in severe cases $[2,3]$.

Mucormycosis is a rapidly progressing fungal infection caused by filamentous fungi in the Mucoraceae family and is frequently seen in diabetic and immunocompromised patients. With onset of second wave of COVID 19 in India, cases of opportunistic fungal infection with mucorale species have increased rapidly. The rhino-orbito-cerebral localization is the most frequent form (44-49\%), followed by the pulmonary and cutaneous localizations (10\%), then the gastrointestinal localization and the disseminated form [5]. The incidence rate of mucormycosis globally varies from 0.005 to 1.7 per million population [6]. Whereas, in Indian population its prevalence is 0.14 per 1000 , which is about 80 times higher than developed countries.[7] The main reason being the second largest population and increasing number of cases of Diabetes Mellitus. Importantly, DM has been the most common risk factor linked with mucormycosis in India, although haematological malignancies and organ transplant takes the lead in Europe and the USA [8].

Rhino-orbital infection usually begins with fungal spores, inhaled and invade the nasal mucosa, and sinusitis develops as the fungus spreads to the paranasal sinuses. Orbital involvement occurs when the infection invades the orbital wall from the paranasal sinuses. Symptoms may include pain, chemosis, vision loss, ophthalmoplegia and proptosis. Ophthalmoplegia arises from infection of the muscles and orbital space or when the third, fourth and sixth cranial nerves are affected. Peripheral seventh cranial nerve paresis or paralysis and hypoesthesia of the face are often observed. $[9,10]$ 


\section{Materials and Methods}

This study was conducted at the department of E.N.T. at PanditDeenDayalUpadhyay Medical College, Churu. Cases presented to E.N.T. OPD with clinical features of invasive rhino-orbital fungal infection for the period of two month from $1^{\text {st }}$ June 2021 were included in the study. Detailed history was taken from all the patients, through examination was done. Patients were then investigated further by nasal endoscopy, HRCT PNS, MRI scans and with $\mathrm{KOH}$ mount and culture of involved tissue. Patient's COVID-19 status was ascertained by clinical history, RT-PCR test and HRCT chest score of the patients. The clinical association of occurrence of invasive fungal infections in post covid and non-covid patients along with other risk factors like diabetes mellitus, immunocompromised state and long term steroid use was done using statistical methods.

\section{Results}

Twenty one patients visited our OPD during the study period with history and clinical features suggestive of rhino orbital invasive mucor mycosis. This is around 320\% increase in number of invasive fungal infection cases as compared to monthly average patients visit due to invasive fungal infection in last 3 years at our institute. Of these cases 15 were male patients and 6 were female patients with male: female ratio of 2.5:1. The age range between 25 years of age to 78 years of age with mean age of 49.62 years. Maximum number of patients was in sixth decade of life with 7 (33.33\%) cases followed by fourth decade with 6 (28.19\%) cases and seventh decade with 5 (19.05\%) cases.

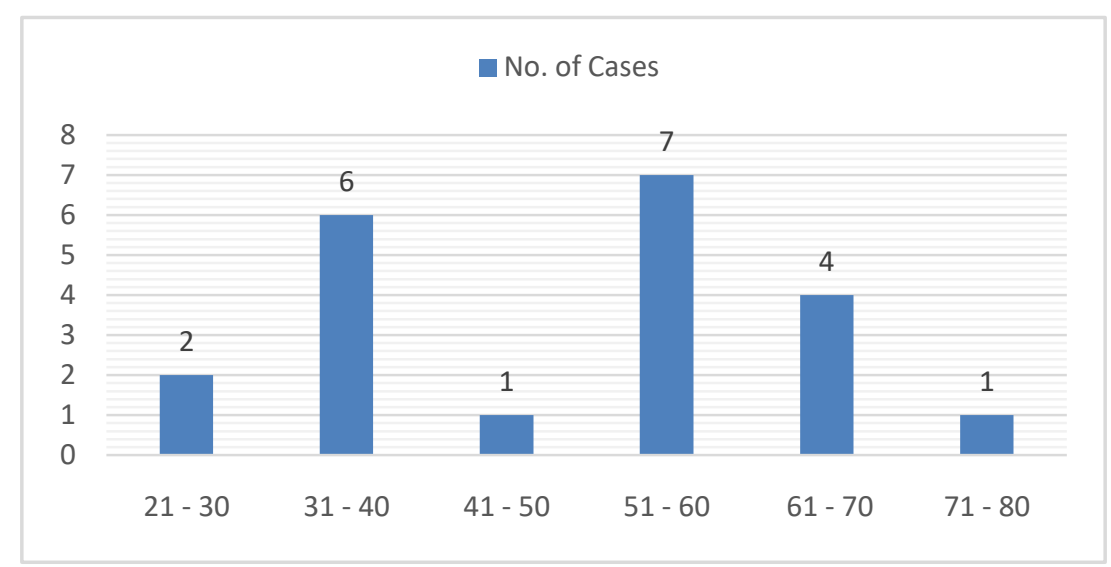

Figure 1: No. of cases in different age groups.

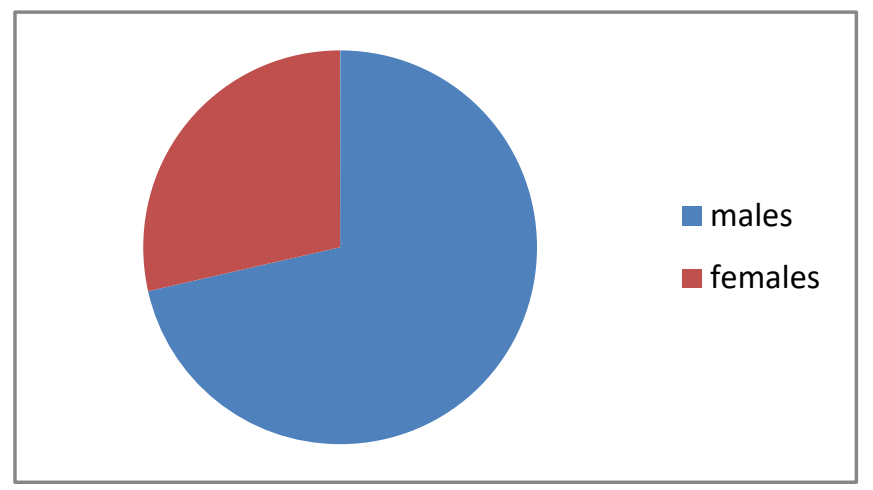

Figure 2: Male, Female ratio

Most common presentation was swelling and pain in the cheek region and red eye and swelling around the eye, three patients presented with maxillary swelling with blackish discoloration of teeth. On ENT examination 8 patients showed crusted blackish lesions in the nasal cavity with hyperaemia and oedema of nasal mucosa, four patients had crusted lesion on hard palate and four patients had swelling and black discoloured lesions on alveolar region of maxilla with oedema and crusting over the oral mucosa. On ophthalmological examination there was chemosis of conjunctiva and oedema of upper and lower eye lids in 7 patients, visual acuity was reduced in affected eye of 7 patients. Proptosis and lateral gaze limitation were observed in 3 patients.

CT and MRI scans were advised. CT showed polypoidal mucosal thickening and hyperdense foci within maxillary and frontal sinuses extending into the nasal cavity in 17 patients and partial sinus wall erosion in 3 patients. Nasal biopsies were taken in all patients, endoscopic biopsies from maxillary sinus were taken from 4 patients and sent for $\mathrm{KOH}$ mount examination and culture. 
Out of the 21 cases with clinical and radiological suspicion of invasive fungal infection (? mucormycosis), $\mathrm{KOH}$ mount examination showed evidence of fungal hyphae in 20 cases.

Sixteen patients (76.19\%) cases gave history for positive RTPCR test for COVID-19 within last 3 months and 4 patients (19.04\%) had negative RT PCR for COVID-19 but had HRCT chest score suggestive of COVID-19 pneumonia, i.e., a total of 20 (95.23\%) patients were post covid status and 1 case (4.77\%) were non covid status. Out of the total diagnosed cases of invasive fungal infection 12 (57.14\%) patients were known cases of diabetes mellitus, 10 patients on oral hypoglycaemics and 2 patients on insulin treatment, all these patients were hyperglycaemic on examination. Nine (42.85\%) cases had newly diagnosed hyperglycaemia with no past history of diabetes mellitus and were not on any kind of treatment for hyperglycaemia. One patient was a diagnosed case of Chronic Myeloid Leukaemia who was on treatment.

Table 1: Presence of risk factors in cases of mucormycosis.

\begin{tabular}{|l|l|l|}
\hline Risk Factors & Post Covid & Non Covid \\
\hline Known case of DM & 11 & 1 \\
\hline New Hyperglycemia & 9 & 0 \\
\hline On chemotherapy for CML & 0 & 1 \\
\hline
\end{tabular}

History of steroid use during the management of COVID19 pneumonia was given by 14 (66.66\%) patients out of the 21 patients.

\section{Discussion}

Mucormycosis is an acute and potentially fatal fungal infection caused by fungi related to the mucoraceae family. It is categorized in rhinocerebral, cutaneous, disseminated, gastrointestinal, or pulmonary.[11] It is most commonly encountered in immuno-compromise patients. Diabetes Mellitus is one of the most common risk factor documented in literature.[12] COVID-19 positive patients also showed multiple co infections. In a recent review, 62/806 (8\%) patients had secondary bacterial or fungal infections during hospital admission. There was widespread use of broadspectrum antibiotics, with as many 1450/2010 (72\%) of patients receiving these drugs, often with no underlying evidence of infection [13].

There are specific pathophysiologic features of COVID-19 that may allow secondary fungal infections, like the immune dysregulation associated with COVID-19, with reduced numbers of $\mathrm{T}$ lymphocytes, CD4+T, and CD8+T cells, may alter innate immunity [14].

During the current second wave of the COVID-19 pandemic in India, an increase in fungal infections, predominantly rhino-orbital mucormycosis, has been documented [15]. Similarly, we encountered a 320\% increase in number of invasive fungal infection cases at our institute. White et al. screened 135 adults with COVID-19 infection and reported an incidence of invasive fungal infections of $26.7 \%$ (commonly aspergillosis (14.1\%), or yeast, usually candida $(12 \cdot 6 \%)$ ). Patients with invasive fungal diseases had higher mortality (53\% with vs $31 \%$ without), which was significantly reduced by appropriate therapy. Corticosteroid therapy and a past history of chronic pulmonary disease were associated with a higher risk of invasive fungal disease [16].

In this study 95\% patients were post COVID-19 status with moderate to severe COVID-19 associated pneumonia and one patient was non COVID-19 status with history of CML for 10 years and was on chemotherapy. Pre-existing diabetes mellitus was there in $57 \%$ cases and $47 \%$ cases showed hyperglycaemia on presentation, that makes more than $95 \%$ patients with either uncontrolled diabetes or new hyperglycaemia. With this in our study we observe significant association of developing diabetes in COVID-19 exposed patients who subsequently developed invasive fungal infection. The use of steroids for the management of COVID pneumonia was present in 66\% patient which indicates that steroid use may also predispose patient to exacerbate previous asymptomatic fungal infections or development of new fungal infection post treatment.

The signs of orbital infection were noticed in lesser number of cases and these appeared later than the nasal infection. All these factors tend to facilitate fungal coinfection, along with any possible COVID-19 pathophysiological mechanisms. In our case, either a previously undiagnosed mucor infection may have been aggravated or it may have subsequently developed.

\section{Conclusion}

COVID-19 is associated with a significant incidence of secondary infections, both bacterial and fungal probably due to immune dysregulation. The widespread use of steroids/monoclonal antibodies/broad-spectrum antibiotics as part of the management protocols against COVID-19 may lead to the exacerbation of preexisting fungal diseases or development of new infection. Treating Physicians should make themselves aware and prepare for the possibility of invasive secondary fungal infections in patients with COVID-19 infection especially in patients with preexisting risk factors and should enable early diagnosis and treatment with the subsequent reduction of mortality and morbidity.

\section{Bibliography}

1. Farnoosh G, Alishiri G, HosseiniZijoud SR, Dorostkar R, JalaliFarahani A. Understanding the severe acute respiratory syndrome coronavirus 2 (SARS-CoV-2) and coronavirus disease (COVID19) based on available evidence-A narrative review. 
J Mil Med. 2020;22:1-11.

2. Lansbury L, Lim B, Baskaran V, Lim WS: Coinfections in people with COVID-19: a systematic review and meta-analysis. SSRN Electron J. 2020, 10.2139/ssrn.3594598

3. Zhu $\mathrm{X}, \mathrm{Ge} \mathrm{Y}, \mathrm{Wu} \mathrm{T}$, et al.: Co-infection with respiratory pathogens among COVID-2019 cases . Virus Res. 2020, 285:198005.

4. Jain VK, Iyengar KP, Vaishya R. Differences between First wave and Second wave of COVID-19 in India. Diabetes MetabSyndr. 2021 May 8:S18714021(21)00147-8. doi: 10.1016/j.dsx.2021.05.009. Epub ahead of print. PMID: 33992554; PMCID: PMC8106236.

5. G. Reid, J.P.L. Iii, M.C. Fishbein, N.M. Clark, Mucormycosis, SeminRespir. Crit.Care Med. 41 (1) (2020) 99-114, févr.

6. Jeong W, Keighley C, Wolfe R, Lee WL, Slavin MA, Kong DC, Chen SA. The epidemiology and clinical manifestations of mucormycosis: a systematic review and meta-analysis of case reports. Clinical Microbiology and Infection. 2019 Jan 1;25(1):26-34.

7. Chander J, Kaur M, Singla N, Punia RP, Singhal SK, Attri AK, Alastruey-Izquierdo A, Stchigel AM, Cano-Lira JF, Guarro J. Mucormycosis: battle with the deadly enemy over a five-year period in India. Journal of Fungi. 2018 Jun;4(2):46.

8. Prakash H, Chakrabarti A. Global epidemiology of mucormycosis. J Fungi 2019;5:26.
9. Roden MM, Zaoutis TE, Buchanan WL, Knudsen TA, Sarkisova TA, Schaufele RL, Sein M, Sein T, Chiou CC, Chu JH, Kontoyiannis DP, Walsh TJ. Epidemiology and outcome of zygomicosis: a review of 929 reported cases. Clin Infect Dis. 2005;41:634653

10. Yohai RA, Bullock JD, Aziz AA, Markert RJ. Survival factors in rhino-orbital-cerebral mucormycosis. SurvOphthalmol. 1994;39:3-22

11. Suganya R, Malathi N, Karthikeyan V, Janagaraj VD: Mucormycosis: a brief review . J Pure ApplMicrobiol. 2019, 13:161-165.

12. Jeong $\mathrm{W}$, Keighley $\mathrm{C}$, Wolfe $\mathrm{R}$, et al.: The epidemiology and clinical manifestations of mucormycosis: a systematic review and metaanalysis of case reports. ClinMicrobiol Infect. 2019, 25:26-34.

13. Rawson TM, Moore LSP, Zhu N, et al. Bacterial and fungal co-infection in individuals with coronavirus: a rapid review to support COVID-19 antimicrobial prescribing. Clin Infect Dis. 2020.

14. Gangneux JP, Bougnoux ME, Dannaoui E, Cornet M, Zahar JR. Invasive fungal diseases during COVID-19: we should be prepared. J Mycol Med. 2020; 30:100971.

15. Dyer O (2021) Covid-19: India sees record deaths as "black fungus" spreads fear. BMJ 373.

16. White PL, Dhillon R, Cordey A, et al. A national strategy to diagnose COVID-19 associated invasive fungal disease in the ICU. Clin Infect Dis. 2020. 